\title{
Informationen der Orthopädischen Gesellschaft firr Osteologie e. V.
}

\section{OGO}

\section{Vorstand}

\section{Präsident}

Prof. Dr. Christopher Niedhart

Orthopädische Gemeinschaftspraxis

Liecker Str. 23

52525 Heinsberg

Tel.: + 49 (0) 2452/9000 10

Fax: + 49 (0) 2452/9000118

E-Mail: cniedhart@gmx.de

\section{Vizepräsident}

Prof. Dr. med. Klaus M. Peters

Chefarzt der Orthopädie und Osteologie

Rhein-Sieg-Klinik

Höhenstraße 30

51588 Nümbrecht

Tel.: + $49(0) 2293 / 920603$

Fax: + $49(0) 22$ 93/920642

E-Mail: kpeters@rhein-sieg-klinik.dbkg.de

\section{Schatzmeister}

Prof. Dr. med. habil. Uwe Maus, MHBA

Leitender Arzt der Klinik für Orthopädie und Spezielle Orthopädische Chirurgie, Universitätsklinik für Orthopädie und Unfallchirurgie

Georgstr. 12

Oldenburg

Tel.: + 49(0) 441/2291570

Fax: $+49(0) 441 / 2291595$

E-Mail: uwe.maus@pius-hospital.de

\section{Schriftführer}

Dr. Dieter Veith

Arzt für Orthopädie

Cornelia-Passage 8

79312 Emmendingen

Tel.: +49(0) 7641/9308760

Fax: $+49(0) 7641 / 9308777$

\section{Mitarbeiterin im OGO- Sekretariat}

Frau Janina Jendritzki

E-Mail: ogo.buero@googlemail.com

\section{Mitgliedsbeitrag}

Der Mitgliedsbeitrag 2018 beträgt für ordentliche Mitglieder Euro 80,-

Der Beitrag schließt den Bezug der Fachzeitschrift Osteologie ein.

OGO im Internet

www.ogo-ev.de 